\title{
A NEW SCALING FOR NEWTON'S ITERATION FOR THE POLAR DECOMPOSITION AND ITS BACKWARD STABILITY*
}

\author{
RALPH BYERS ${ }^{\dagger}$ AND HONGGUO XU
}

\begin{abstract}
We propose a scaling scheme for Newton's iteration for calculating the polar decomposition. The scaling factors are generated by a simple scalar iteration in which the initial value depends only on estimates of the extreme singular values of the original matrix, which can, for example, be the Frobenius norms of the matrix and its inverse. In exact arithmetic, for matrices with condition number no greater than $10^{16}$, with this scaling scheme no more than 9 iterations are needed for convergence to the unitary polar factor with a convergence tolerance roughly equal to $10^{-16}$. It is proved that if matrix inverses computed in finite precision arithmetic satisfy a backward-forward error model, then the numerical method is backward stable. It is also proved that Newton's method with Higham's scaling or with Frobenius norm scaling is backward stable.
\end{abstract}

Key words. matrix sign function, polar decomposition, singular value decomposition (SVD), Newton's method, numerical stability, scaling

AMS subject classifications. 65F05, 65G05

DOI. $10.1137 / 070699895$

1. Introduction. Every matrix $A \in \mathbb{C}^{n \times n}$ has a polar decomposition $A=Q H$, where $H=H^{*} \in \mathbb{C}^{n \times n}$ is Hermitian positive semidefinite and $Q \in \mathbb{C}^{n \times n}$ is unitary, i.e., $Q^{*} Q=I$. The polar decomposition is unique with positive definite symmetric factor $H$ iff $A$ is nonsingular. Its applications include unitary approximation and distance calculations $[8,9,12]$. The polar decomposition generalizes to rectangular matrices; see, for example, [15]. We consider only the square matrix case here, because numerical methods for computing the polar decomposition typically begin by reducing the problem down to the square matrix case using, for example, a QR factorization $[5,8]$. (An algorithm that works directly with rectangular matrices appears in [6].)

The polar decomposition may be easily constructed from a singular value decomposition (SVD) of $A$. However, the SVD is a substantial calculation that displays much more of the structure of $A$ than does the polar decomposition. Constructing the polar decomposition from the SVD destroys this extra information and wastes the arithmetic work used to compute it. It is intuitively more appealing to use the polar decomposition as a preliminary step in the computation of the SVD as in [12].

When $A$ is nonsingular, one way to compute the polar decomposition is through Newton's iteration:

$$
Q_{k+1}=\frac{1}{2}\left(\zeta_{k} Q_{k}+\left(\zeta_{k} Q_{k}\right)^{-*}\right), \quad Q_{0}=A,
$$

where $\zeta_{k}=\zeta\left(Q_{k}\right)>0$ is a positive scalar function of $Q_{k}$ chosen to accelerate convergence [8]. Each iterate $Q_{k}$ has polar decomposition $Q_{k}=Q H_{k}$, where $Q$ is

${ }^{*}$ Received by the editors August 13, 2007; accepted for publication (in revised form) by N. J. Higham April 24, 2008; published electronically September 4, 2008.

http://www.siam.org/journals/simax/30-2/69989.html

${ }^{\dagger}$ This author is deceased. Former address: Department of Mathematics, University of Kansas, Lawrence, KS 66045. This material was based upon work partially supported by the University of Kansas General Research Fund allocations 2301062-003 and 2301054-003 and by the NSF awards 0098150, 0112375, and 9977352.

${ }^{\ddagger}$ Department of Mathematics, University of Kansas, Lawrence, KS 66045 (xu@math.ku.edu). This author was partially supported by NSF grant EPS-9874732 with matching support from the State of Kansas and the University of Kansas General Research Fund allocation 2301717-003.

822 
the unitary polar factor of $A, H_{0}=H$ is the Hermitian polar factor of $A$, and $H_{k+1}=\left(\zeta_{k} H_{k}+\left(\zeta_{k} H_{k}\right)^{-1}\right) / 2, k \geq 0$. For appropriately chosen acceleration parameters $\zeta_{k}, \lim _{k \rightarrow \infty} H_{k}=I$. Hence, the unitary polar factor is $Q=\lim _{k \rightarrow \infty} Q_{k}$, and the Hermitian polar factor is $H=\lim _{k \rightarrow \infty} Q_{k}^{*} A$.

Iteration (1.1) was first proposed in [8] and studied further in $[5,6,14]$. It is called "Newton's iteration" because it can be derived from Newton's method applied to the equation $X^{*} X=I$. It is closely related to Newton's iteration for the matrix sign function $[17,22]$.

Simplicity is an attractive feature of (1.1). Apart from the computation of $\zeta_{k}$, each iteration needs only one matrix inversion and one matrix-matrix addition. The simplicity allows implementations of (1.1) to take advantage of the hierarchical memory and parallelism $[1,2,13]$. Many authors have studied choices of the acceleration parameters $\zeta_{k}[3,4,8,16,17,22]$. If $\zeta_{k} \equiv 1$, then the iterates $Q_{k}$ converge quadratically to the unitary polar factor $Q$ [8]. Convergence is also quadratic if $\zeta=\zeta(U)$ is a smooth function of $U \in \mathbb{C}^{n \times n}$ and $\zeta(U)=1$ whenever $U$ is unitary.

The choice

$$
\zeta_{k}^{(2)}=\sqrt{\frac{\left\|Q_{k}^{-1}\right\|_{2}}{\left\|Q_{k}\right\|_{2}}},
$$

where $\|\cdot\|_{2}$ is the spectral norm is proposed in [8]. This scale factor is optimal in the sense that, given $Q_{k},(1.2)$ minimizes the next error $\left\|Q_{k+1}-Q\right\|_{2}$. With this scale factor, for the matrices $Q_{k}$ generated by (1.1), the error sequence $\left\|Q_{k}-Q\right\|_{2}$ converges monotonically to zero. Unfortunately, to determine the scale factor (1.2), one needs to compute two extreme singular values of $Q_{k}$ at each iteration. In order to preserve the rapid convergence of (1.1) with scaling (1.2), the highly accurate values of these extreme singular values are required to guarantee $\zeta_{k}^{(2)} \rightarrow 1$. This is expensive enough to make scale factor (1.2) unattractive.

To save the cost of computing the extreme singular values, one might approximate (1.2). A commonly used scale factor is the $(1, \infty)$-scaling

$$
\zeta_{k}^{(1, \infty)}=\left(\frac{\left\|Q_{k}^{-1}\right\|_{1}\left\|Q_{k}^{-1}\right\|_{\infty}}{\left\|Q_{k}\right\|_{1}\left\|Q_{k}\right\|_{\infty}}\right)^{\frac{1}{4}},
$$

(where $\|\cdot\|_{1}$ and $\|\cdot\|_{\infty}$ are the 1-norm and $\infty$-norm, respectively) which was proposed by Higham in [8]. The factor $\zeta_{k}^{(1, \infty)}$ is within a constant factor of $\zeta_{k}^{(2)}$. It adds a negligible amount of arithmetic work compared to the cost of $Q_{k}^{-1}$, which is needed at each iteration anyway.

The scale factor

$$
\zeta_{k}^{(F)}=\left\|Q_{k}^{-1}\right\|_{F}^{1 / 2}\left\|Q_{k}\right\|_{F}^{-1 / 2},
$$

(where $\|\cdot\|_{F}$ is the Frobenius norm) is discussed in $[5,8,16]$. It can also be computed at a negligible cost. It is optimal in the sense that, given $Q_{k}$, it minimizes $\left\|Q_{k+1}\right\|_{F}$ and causes the sequence $\left\|Q_{k}\right\|_{F}$ to converge monotonically [5].

Another relatively inexpensive scale factor is [4]

$$
\zeta_{k}^{(d)}=\left|\operatorname{det}\left(Q_{k}\right)\right|^{-1 / n}
$$

The complex modulus of the determinant is very inexpensively obtained from the same matrix factorization used to calculate $Q_{k}^{-1}$. This scaling is optimal in the sense 
that, for a given iterate $Q_{k}$, it minimizes $D\left(Q_{k+1}\right)=\sum_{j=1}^{n}\left(\ln \left(\sigma_{j}^{(k+1)}\right)\right)^{2}$, where $\sigma_{j}^{(k+1)}$ is the $j$ th singular value of $Q_{k+1}$. The function $D\left(Q_{k+1}\right)$ is a measure of the departure of $Q_{k+1}$ from the unitary matrices.

This paper considers the suboptimal scaling strategy

$$
\zeta_{0}=1 / \sqrt{a b}, \quad \zeta_{1}=\sqrt{\frac{2 \sqrt{a b}}{a+b}}, \quad \zeta_{k}=1 / \sqrt{\rho\left(\zeta_{k-1}\right)}, \quad k=2,3, \ldots,
$$

where $\rho(x)=\left(x+x^{-1}\right) / 2$ and $a$ and $b$ are any numbers such that $0<a \leq\left\|A^{-1}\right\|_{2}^{-1} \leq$ $\|A\|_{2} \leq b$. Apart from estimating the extreme singular values of the initial matrix $Q_{0}=A$, the scale factor costs only several floating point operations per iteration. Moreover, only the rough estimates of $\|A\|_{2}$ and $\left\|A^{-1}\right\|_{2}^{-1}$ are needed. One may simply choose $a=\left\|A^{-1}\right\|_{F}^{-1}$ and $b=\|A\|_{F}$. From Table 2.1 with such choices for any matrices with condition number no greater than $10^{16}$ and size no greater than $10^{11}$, at most nine iterations of (1.1) with scaling (1.6) are necessary to approximate the unitary polar factor $Q$ to within 2-norm distance less than $10^{-16}$.

We show below that, in the presence of rounding error, (1.1) with (1.6) is numerically stable assuming that matrix inverses are calculated with small forward-backward error. This is the case, for example, when matrix inverses are computed using the bidiagonal reduction [7, p. 252]. We also prove the numerical stability of Newton's iteration with any of the scalings (1.2)-(1.4).

Commenting on an early draft of this paper, Ziętak pointed out that the suboptimal (quasi-optimal) scaling parameters were discovered independently by Kiełbasiński but not published in the open literature. They were presented by Ziętak at the 1999 Householder meeting at Whistler and the 1999 ILAS conference at Barcelona. In section 5 of their recent paper [19], Kiełbasiński, Zieliński, and Ziętak mention the quasi-optimal scaling parameters. In [18], these authors gave an error analysis of Higham's method [8] with the same mixed backward-forward stability assumption for matrix inversion. They gave numerical experiments in [23].

In the following $A \in \mathbb{C}^{n \times n}$ is always nonsingular. $A=U \Sigma V^{*}$ is the SVD of $A$, where $U$ and $V$ are unitary, $\Sigma=\operatorname{diag}\left(\sigma_{1}, \cdots, \sigma_{n}\right)$ is diagonal, and $\sigma_{1} \geq \cdots \geq \sigma_{n} \geq 0$ are the singular values of $A$. The set of the singular values is denoted by $\sigma(A)$. The condition number with respect to the spectral norm of $A$ is denoted by $\kappa_{2}(A)=\sigma_{1} / \sigma_{n}$. Following $[7$, p. 18], a flop is the computational work of a floating point addition, subtraction, multiplication, or division together with the associated subscripting and indexing overhead. It takes two flops to execute the Fortran statement $A(I, J)=$ $A(I, J)+C * A(K, J)$.

2. Scaling and convergence. Let $A \in \mathbb{C}^{n \times n}$ be nonsingular, with the SVD $A=U \Sigma V^{*}$, with $\Sigma=\operatorname{diag}\left(\sigma_{1}, \sigma_{2}, \ldots, \sigma_{n}\right)$. Each Newton iterate $Q_{k}$ in (1.1) has the $\operatorname{SVD} Q_{k}=U \Sigma_{k} V^{*}$, where

$$
\Sigma_{k+1}=\left(\zeta_{k} \Sigma_{k}+\left(\zeta_{k} \Sigma_{k}\right)^{-1}\right) / 2, \quad \Sigma_{0}=\Sigma .
$$

In particular, $Q_{k}$ has singular values $\sigma_{1}^{(k)}, \sigma_{2}^{(k)}, \ldots \sigma_{n}^{(k)}$ (in no particular order when $k>0$ ) that obey

$$
\sigma_{j}^{(0)}=\sigma_{j}, \quad \sigma_{j}^{(k+1)}=\frac{1}{2}\left(\zeta_{k} \sigma_{j}^{(k)}+\frac{1}{\zeta_{k} \sigma_{j}^{(k)}}\right)=\rho\left(\zeta_{k} \sigma_{j}^{(k)}\right), \quad k=0,1,2, \ldots,
$$


where $\rho(x)=\left(x+x^{-1}\right) / 2$. For appropriately chosen $\zeta_{k}, \lim _{k \rightarrow \infty} \sigma_{j}^{(k)}=1$, and $\lim _{k \rightarrow \infty} Q_{k}=U V^{*}=Q$. Consequently, the convergence properties of (1.1) derive directly from the $n$ scalar sequences $\sigma_{j}^{(k)}$ determined by (2.2). To attain good convergence behavior in (1.1), the acceleration parameters $\zeta_{k}$ must interact well with $\rho(x)=\left(x+x^{-1}\right) / 2$.

The following two lemmas list some easily verified elementary properties of $\rho(x)=$ $\left(x+x^{-1}\right) / 2$.

LEMMA 2.1. If $x>0$, then

1. $\rho\left(\frac{1}{x}\right)=\rho(x)$.

2. $1 \leq \rho(x) \leq \max \left(x, x^{-1}\right)$, with either equality iff $x=1$.

3. $\rho(x)$ is decreasing on $x \in(0,1]$ and increasing on $x \in[1, \infty)$.

Lemma 2.2. Suppose that $0<a \leq b$. Define $\alpha_{\zeta}=\max \left\{(\zeta a)^{-1}, \zeta b\right\}$, and $\zeta_{\text {opt }}=(a b)^{-1 / 2}$. Then we have the following properties.

1. For any $\zeta>0,1 \leq \max _{a<x<b} \rho(\zeta x)=\rho\left(\alpha_{\zeta}\right)$, and $1=\max _{a<x<b} \rho(\zeta x)$ iff $\alpha_{\zeta}=1$.

2. For any $\zeta>0,1 \leq \min _{a<x<b} \rho(\zeta x)$, and $1=\min _{a<x<b} \rho(\zeta x)$ iff $\zeta a \leq 1 \leq \zeta b$.

3. $\min _{\zeta>0} \alpha_{\zeta}=\alpha_{\zeta_{\text {opt }}}=\sqrt{b / a}$, and $\zeta=\zeta_{\text {opt }}$ is the only minimizer.

4. $\min _{\zeta>0} \max _{a \leq x \leq b} \rho(\zeta x)=\min _{\zeta>0} \rho\left(\alpha_{\zeta}\right)=\rho\left(\alpha_{\zeta_{\text {opt }}}\right)=\rho(\sqrt{b / a})$.

In the following, for ease of notation let $\tau(x)$ be the function

$$
\tau(x)=\rho(\sqrt{x})=\frac{1}{2}\left(\sqrt{x}+\frac{1}{\sqrt{x}}\right) .
$$

The $k$-fold composition of $\tau(x)$ with itself is written $\tau^{k}(x)$, i.e., $\tau^{0}(x)=x, \tau^{1}(x)=$ $\tau(x)$, and for $k>1, \tau^{k+1}(x)=\tau\left(\tau^{k}(x)\right)$. Similarly $\rho^{k}(x)$ is the $k$-fold composition of $\rho(x)=\left(x+x^{-1}\right) / 2$ with itself.

Suppose that $0<a \leq \sigma_{n} \leq \sigma_{1} \leq b$. Consider the sequence of intervals generated by Newton's iteration: $\left[a_{0}, b_{0}\right]=[a, b],\left[a_{1}, b_{1}\right]=\rho\left(\zeta_{0}\left[a_{0}, b_{0}\right]\right),\left[a_{2}, b_{2}\right]=\rho\left(\zeta_{1}\left[a_{1}, b_{1}\right]\right)$, .... It follows from $(2.2)$ that $\sigma_{j}^{(k)} \in\left[a_{k}, b_{k}\right], j=1,2, \ldots, n, k=0,1, \ldots$ Note that $\min _{x>0} \rho(x)=1$, so for $k \geq 1,\left[a_{k}, b_{k}\right] \subseteq\left[1, b_{k}\right]$ and

$$
1 \leq \sigma_{j}^{(k)} \leq b_{k} .
$$

It is intuitively satisfying to choose the sequence of acceleration parameters $\zeta_{k}$ in (1.1) to minimize the sequence $b_{k}$.

From Lemma 2.2, the initial optimal scaling factor is $\zeta_{0}=(a b)^{-1 / 2}$. The initial interval is scaled to be $\zeta_{0}[a, b]=[\sqrt{a / b}, \sqrt{b / a}]$ which contains 1 . The next interval is

$$
\left[a_{1}, b_{1}\right]=\rho\left(\zeta_{0}[a, b]\right)=[1, \rho(\sqrt{b / a})]=[1, \tau(b / a)],
$$

where $\tau(x)$ is given by (2.3). The left endpoint is $a_{1}=1$, so the optimal scaling factor for the next iteration is $\zeta_{1}=b_{1}^{-1 / 2}=1 / \sqrt{\tau(b / a)}$. The next interval is

$$
\left[a_{2}, b_{2}\right]=\rho\left(\zeta_{1}\left[a_{1}, b_{1}\right]\right)=[1, \rho(\sqrt{\tau(b / a)})]=\left[1, \tau^{2}(b / a)\right] .
$$

An easy induction shows that the sequence of intervals is $\left[a_{0}, b_{0}\right]=[a, b]$ and for $k \geq 1,\left[a_{k}, b_{k}\right]=\left[1, \tau^{k}(b / a)\right]$, and the sequence of optimal scaling factors is

$$
\zeta_{0}=1 / \sqrt{a b}, \quad \zeta_{k}=1 / \sqrt{\tau^{k}(b / a)}, \quad k=1,2,3, \ldots,
$$

which is equivalent to (1.6). 
Since $\tau(x)=\rho(\sqrt{x}) \leq \rho(x)$ for $x \geq 1$,

$$
\tau(b / a) \leq \rho(b / a) .
$$

By induction we have

$$
\tau^{k}(b / a) \leq \rho^{k}(b / a)
$$

for all $k \geq 1$. The sequence $b / a, \rho(b / a), \rho^{2}(b / a), \ldots$, is generated by Newton's iteration $x_{k+1}=\rho\left(x_{k}\right)$, with $x_{0}=b / a$. It converges to 1 quadratically. Obviously $\tau^{k}(b / a) \geq 1$ for $k \geq 0$. So $b / a, \tau(b / a), \tau^{2}(b / a), \ldots$ also converges to 1 at least quadratically. It is not difficult to show that $1 \leq \tau(x) \leq x$ for any $x \geq 1$. We have

$$
b_{k}=\tau^{k}(b / a)=\tau\left(\tau^{k-1}(b / a)\right) \leq \tau^{k-1}(b / a)=b_{k-1} .
$$

Hence, after the first step, the sequence of intervals satisfies

$$
\left[a_{1}, b_{1}\right] \supseteq\left[a_{2}, b_{2}\right] \supseteq \cdots \supseteq\left[a_{k}, b_{k}\right] \supseteq \cdots,
$$

and it converges to the single point 1 quadratically. (Note $a_{k}=1$ for all $k \geq 1$.) The initial interval, $\left[a_{0}, b_{0}\right]=[a, b]$ is an exception, because, in general, $[a, b] \nsupseteq[1, \tau(b / a)]$.

Based on this fact and (2.4), the convergence properties of (1.1) with (1.6) are clear, and we summarize them in the following theorem.

THEOREM 2.3. If

$$
0<a \leq\left\|A^{-1}\right\|_{2}^{-1} \leq\|A\|_{2} \leq b
$$

and $Q_{k}$ is obtained from the Newton iteration (1.1) with scaling (1.6), then

$$
\left\|Q_{k}-Q\right\| \leq \tau^{k}(b / a)-1 \leq \rho^{k}(b / a)-1, \quad k=1,2, \ldots .
$$

In fact the convergence properties are highly satisfactory even when $b / a$ is large. Table 2.1 uses Theorem 2.3 to list the number of Newton's iteration (1.1) with scaling (1.6) (and exact arithmetic) required to guarantee selected absolute errors $\delta>\left\|Q_{k}-Q\right\|_{2}$ and values of $b / a$. The table demonstrates that Newton's iteration (1.1) with scaling (1.6) typically needs no more than nine iterations to attain typical floating point precision accuracy. The table also demonstrates that convergence is insensitive to the choice of $a$ and $b$-widely differing values of $b / a$ need similar numbers of iterations to attain similar accuracy. In particular the easy-to-compute choices $a=\left\|A^{-1}\right\|_{F}^{-1}$ and $b=\|A\|_{F}$ satisfy (2.6) and are unlikely to lead to even one more iteration than the optimum choices of $a=\left\|A^{-1}\right\|_{2}^{-1}$ and $b=\|A\|_{2}$, particularly for ill-conditioned matrices. For instance, for any $A \in \mathbb{C}^{n \times n}$ with $\kappa_{2}(A)=10^{16}$, for $a=\left\|A^{-1}\right\|_{F}^{-1}$ and $b=\|A\|_{F}$, we have $b / a \leq n \kappa(A)=n 10^{16}$. Then $b / a \leq 10^{27}$ for any $n \leq 10^{11}$, and the number of iterations is 9 , the same as with the optimum choices.

In Theorem 2.3, smaller values of $b / a$ give smaller values of $\tau^{k}(b / a)$ and hence better error bounds. Inequality (2.6) implies that $b / a \geq \kappa_{2}(A)=\left\|A^{-1}\right\|_{2}\|A\|_{2}$, and equality can be achieved only with $a=\left\|A^{-1}\right\|_{2}^{-1}=\sigma_{n}$ and $b=\|A\|_{2}=\sigma_{1}$. With $a=\sigma_{n}$ and $b=\sigma_{1}$, the scaling factors (1.6) are

$$
\zeta_{0}=1 / \sqrt{\sigma_{1} \sigma_{n}}, \quad \zeta_{k}=1 / \sqrt{\tau^{k}\left(\kappa_{2}(A)\right)}, \quad(k \geq 1),
$$

and the corresponding intervals are $\left[\sigma_{n}, \sigma_{1}\right]$, and $\left[1, \tau^{k}\left(\kappa_{2}(A)\right)\right]$ for $k \geq 1$. Let $\Sigma_{k}$ be the matrices generated by (2.1), with $a=\sigma_{n}$ and $b=\sigma_{1}$. It is easy to verify that in 
TABLE 2.1

The number of Newton iterations (1.1) with scaling (1.6) (and exact arithmetic) required to guarantee absolute error $\left\|Q_{k}-Q\right\|_{2}<\delta$ for selected values of $\delta$ and $b / a$ such that $0<a \leq\left\|A^{-1}\right\|_{2}^{-1} \leq$ $\|A\|_{2} \leq$ b. See Theorem 2.3 .

\begin{tabular}{|l|ccccccc|}
\hline$\delta \backslash b / a$ & 10 & $10^{5}$ & $10^{10}$ & $10^{15}$ & $10^{20}$ & $10^{25}$ & $10^{27}$ \\
\hline $10^{-1}$ & 2 & 4 & 5 & 6 & 6 & 6 & 6 \\
\hline $10^{-4}$ & 4 & 5 & 6 & 7 & 7 & 8 & 8 \\
\hline $10^{-7}$ & 4 & 6 & 7 & 8 & 8 & 8 & 8 \\
\hline $10^{-10}$ & 5 & 7 & 7 & 8 & 8 & 9 & 9 \\
\hline $10^{-13}$ & 5 & 7 & 8 & 8 & 9 & 9 & 9 \\
\hline $10^{-16}$ & 5 & 7 & 8 & 9 & 9 & 9 & 9 \\
\hline $10^{-19}$ & 6 & 7 & 8 & 9 & 9 & 10 & 10 \\
\hline
\end{tabular}

this case, the right endpoint of the $k$ th interval is a singular value of $\Sigma_{k}$. This in turn implies that inequality (2.7) is an equality, i.e.,

$$
\left\|Q_{k}-Q\right\|_{2}=\left\|\Sigma_{k}-I\right\|_{2}=\tau^{k}\left(\kappa_{2}(A)\right)-1 .
$$

The number sequence $b_{k}$ was also derived in [16] in order to show the convergence behavior of Newton's method with the optimal scale factors. It is shown that when $a=\sigma_{n}$ and $b=\sigma_{1}$, for $Q_{k}$ generated with $\zeta_{k-1}^{(2)}$ defined in (1.2), one has $\left\|Q_{k}\right\|_{2} \leq b_{k}$ $[16,11]$. Due to this fact we call $\zeta_{k}$ defined in (2.5) suboptimal scale factors. Note that $b_{k}$ is derived based on different interpretations here. It is the right endpoint of the $k$ th interval generated by applying Newton's iteration to the initial interval $[a, b]$. For this interval iteration, $\zeta_{k}$ is the scale factor that minimizes $b_{k+1}-1$ (i.e., it makes $\left[a_{k+1}, b_{k+1}\right]$ as close to 1 as possible).

3. The algorithm. The Newton's method (1.1) with scaling scheme (1.6) is implemented by the following algorithm.

Algorithm 3.1 (Newton's method (1.1) with scaling (1.6)).

Input: Nonsingular matrix $A \in \mathbb{C}^{n \times n}$ and a stopping criterion $\delta>0$.

Output: The polar decomposition $A=Q H$.

Step 0: $\quad$ a. Set $Q_{0}=A$; Compute $Q_{0}^{-*}$

b. Choose $a \leq\left\|Q_{0}^{-1}\right\|_{2}^{-1}$ and $b \geq\left\|Q_{0}\right\|_{2} ; \zeta_{0}=1 / \sqrt{a b}$

c. Set $k=0$

Step 1: While $\left\|Q_{k}-Q_{k}^{-*}\right\|_{F} \geq \delta$

a. $Q_{k+1}=\left(\zeta_{k} Q_{k}+\zeta_{k}^{-1} Q_{k}^{-*}\right) / 2$

b.

$$
\text { If } k=0, \zeta_{1}=\sqrt{\frac{2}{\sqrt{b / a}+\sqrt{a / b}}}
$$

Else $\quad \zeta_{k+1}=\sqrt{\frac{2}{\zeta_{k}^{-1}+\zeta_{k}}}$

End if

c. Compute $Q_{k+1}^{-*}$

d. $k=k+1$

End while

Step 2: $Q=\left(Q_{k}+Q_{k}^{-*}\right) / 2 ; \quad H=\frac{1}{2}\left(Q^{*} A+\left(Q^{*} A\right)^{*}\right)$

Here are some remarks.

1. The matrix $A^{-1}=Q_{0}^{-1}$ needs to be computed in the first iteration anyway. Hence, power iterations on $A$ and $A^{-1}$ may be used evaluate the extreme singular values $\sigma_{1}$ and $\sigma_{n}^{-1}$, respectively, using only $O\left(n^{2}\right)$ extra flops per iteration. These 
estimates may then serve as the suboptimal scaling factors $b$ and $a^{-1}$, respectively. Since highly accurate estimates of $\sigma_{1}$ and $\sigma_{n}$ are unnecessary, a few power iterations should suffice. Alternatively, $\|A\|_{F}$ and $\left\|A^{-1}\right\|_{F}^{-1}$ may be used for $b$ and $a$.

2. The stopping criterion $\left\|Q_{k}-Q_{k}^{-*}\right\|_{F}<\delta$ is essentially equivalent to $\| Q_{k+1}-$ $Q_{k} \|_{F}<\delta$, which is used in [11, section 8.9]. This follows from the fact that when $\zeta_{k} \approx 1$ (which is usually the case for a small $\delta$ ),

$$
Q_{k+1}-Q_{k}=\frac{1}{2}\left(\zeta_{k}^{-1} Q_{k}^{-*}-\left(2-\zeta_{k}\right) Q_{k}\right) \approx \frac{1}{2}\left(Q_{k}^{-*}-Q_{k}\right) .
$$

In practice, in order for the computed $Q_{k}$ to be within $O(\varepsilon)$ of a unitary matrix, where $\varepsilon$ is the machine epsilon, it is sufficient to choose $\delta=O(\sqrt{\varepsilon})$. See (4.16).

3. Commonly the matrix inversion method used in the algorithm is an LU factorization-based method such as the Gaussian elimination with partial pivoting or complete pivoting [8]. Such an inversion method usually works well in practice [18]. In order to guarantee the algorithm to be numerically backward stable, one may use the more expensive bidiagonal reduction-based matrix inversion method provided in Appendix A.1. So the computed matrix inverses satisfy the backward-forward error model. See Assumption 4.1 in section 4 below.

4. Estimating $\sigma_{1}$ and $\sigma_{n}$ usually uses $O\left(n^{2}\right)$ flops. Each iteration uses $2 n^{3}$ flops for the matrix inverse by an LU factorization-based method and $O\left(n^{2}\right)$ flops for matrix addition. Computing $H$ uses $2 n^{3}$ flops. If $p$ is the number of iterations for convergence, then the algorithm uses a total of roughly $2(p+1) n^{3}$ flops [8]. When $p=9$ it is about $20 n^{3}$ flops, which is less than the QR-like SVD method (which takes $22 n^{3}$ to $26 n^{3}$ flops for the SVD and $4 n^{3}$ for $Q$ and $H$ ). If the bidiagonal reduction-based matrix inversion method is used, the total cost will be $2(3 p+1) n^{3}$ flops.

5 . In order to reduce the cost while maintaining numerical stability, one may first use the bidiagonal reduction-based method for a few iterations. When $\kappa_{2}\left(Q_{k}\right)$ is not too large, say 100, one shifts to an LU factorization-based inversion method for the subsequent iterations. The matrix inverses essentially satisfy the backward-forward error model in the latter case [10, section 14]. Also, it takes only a few iterations for the condition number to drop below 100. In the case when $\kappa_{2}(A)=10^{-16}$, with $a=\sigma_{n}$ and $b=\sigma_{1}$, then $\left\|Q_{3}\right\|_{2}=\tau^{3}\left(10^{-16}\right) \approx 42$. Since this is usually the worst case in practice, the bidiagonal reduction-based method is required in no more than 3 iterations. With this strategy, the maximum cost (with $p=9$ ) is $3 \cdot 6 n^{3}+(9-3)$. $2 n^{3}+2 n^{3}=32 n^{3}$ flops.

6. Although the cost for computing the scale factors (1.3)-(1.5) is negligible in Newton's iteration, computing the suboptimal scale factors is essentially costless. Also, the use of suboptimal scaling simplifies the algorithm, since the "shifting scale factor to 1" strategy, which is used for the $(1, \infty)$-scaling $([8])$, is not needed. Finally, with suboptimal scaling, in general, the number of iterations is no greater than 9 , and it can be obtained by simply computing $\tau^{k}(b / a)-1$. It is still not clear how to predict the number of iterations with other scalings, although in practice it is observed that the $(1, \infty)$-scaling and the suboptimal scaling essentially have the same convergence rate.

4. Stability and rounding error analysis. In this section, a first order error analysis establishes that Newton's method (1.1) with scaling (1.6) can be implemented in a backward stable way. The same conclusion is drawn for the scalings (1.2), (1.3), and (1.4). In outline, the approach is to estimate the residual $\|A-\widehat{Q} \widehat{H}\|_{2}$ for the rounding-error-perturbed unitary factor $\widehat{Q}$ and Hermitian factor $\widehat{H}$ produced by finite 
precision arithmetic in the algorithm in section 3. The method here is to first estimate the forward errors $\widehat{Q}-Q$ and $\widehat{H}-H$ and then use them to estimate the residual.

For the error analysis, we employ the standard model of floating point arithmetic with machine epsilon $\varepsilon$ [10, section 2.2].

We also need the following assumptions.

Assumption 4.1. If a nonsingular matrix $A \in \mathbb{C}^{n \times n}$ is inverted using finite precision arithmetic with machine epsilon $\varepsilon$ to obtain a "computed inverse" $X$, then

$$
X=(A+E)^{-1}+F,
$$

where $E, F \in \mathbb{C}^{n \times n}$ are perturbation matrices satisfying

$$
\|E\|_{2} \leq c_{1}(n) \varepsilon\|A\|_{2}, \quad\|F\|_{2} \leq c_{2}(n) \varepsilon\left\|A^{-1}\right\|_{2},
$$

and $c_{i}(n)(i=1,2)$ are some low-degree polynomials of $n$.

In Newton's method it is typical to use Gaussian elimination with partial or complete pivoting for computing matrix inverses. Although it works well in practice, the computed matrix inverses may not satisfy Assumption 4.1 [10, section 14.1]. We show in Appendix A.1 that Assumption 4.1 is satisfied by a matrix inversion algorithm that uses the bidiagonal reduction method.

Assumption 4.2.

$$
c_{3}(n) \kappa_{2}(A) \varepsilon<1,
$$

where $c_{3}(n)$ is a low-degree polynomial of $n$.

Note that $1 / \kappa_{2}(A)$ is the measure of the relative distance of a nonsingular $A$ to the nearest singular matrices $[7$, p. 73$]$, i.e.,

$$
\frac{1}{\kappa_{2}(A)}=\min _{\operatorname{det}(A+E)=0} \frac{\|E\|_{2}}{\|A\|_{2}}
$$

If such a condition doesn't hold, then matrices like $A+E$ in Assumption 4.1 can be singular. So this is a condition about the numerical nonsingularity of $A$. It is essential in the subsequent first order error analysis, although it won't be explicitly stated.

We now begin the error analysis. In practice, rounding errors perturb Newton's method recurrence (1.1). Under Assumptions 4.1, if $\widehat{Q}_{k}$ is the computed version of $Q_{k}$, then

$$
\begin{aligned}
\widehat{Q}_{k+1} & =\frac{\zeta_{k}}{2} \widehat{Q}_{k}+F_{k, 1}+\frac{1}{2 \zeta_{k}}\left(\left(\widehat{Q}_{k}+F_{k, 2}\right)^{-*}+F_{k, 3}\right) \\
& =\frac{\zeta_{k}}{2}\left(\widehat{Q}_{k}+F_{k, 2}\right)+\frac{1}{2 \zeta_{k}}\left(\widehat{Q}_{k}+F_{k, 2}\right)^{-*}+\left(F_{k, 1}+\frac{1}{2 \zeta_{k}} F_{k, 3}-\frac{\zeta_{k}}{2} F_{k, 2}\right) \\
& =: \frac{\zeta_{k}}{2}\left(\widehat{Q}_{k}+F_{k b}\right)+\frac{1}{2 \zeta_{k}}\left(\widehat{Q}_{k}+F_{k b}\right)^{-*}+F_{k f},
\end{aligned}
$$

where $F_{k b}=F_{k, 2}$ and $F_{k f}=\left(F_{k, 1}+\frac{1}{2 \zeta_{k}} F_{k, 3}-\frac{\zeta_{k}}{2} F_{k, 2}\right)$. The perturbation matrix $F_{k, 1}$ represents rounding errors introduced by floating point matrix addition and scalar multiplication, and the perturbation matrices $F_{k, 2}$ and $F_{k, 3}$ represent rounding errors 
introduced by matrix inversion under Assumption 4.1. The F's obey the bounds

$$
\begin{aligned}
\left\|F_{k, 1}\right\|_{2} & \leq d_{1} \varepsilon \max \left(\left\|\zeta_{k} \widehat{Q}_{k}\right\|_{2},\left\|\zeta_{k}^{-1} \widehat{Q}_{k}^{-*}\right\|_{2}\right) \\
\left\|F_{k, 2}\right\|_{2} & \leq d_{2} \varepsilon\left\|\widehat{Q}_{k}\right\|_{2} \\
\left\|F_{k, 3}\right\|_{2} & \leq d_{3} \varepsilon\left\|\widehat{Q}_{k}^{-*}\right\|_{2} \\
\left\|F_{k b}\right\|_{2} & \leq d_{b} \varepsilon\left\|\widehat{Q}_{k}\right\|_{2} \\
\left\|F_{k f}\right\|_{2} & \leq d_{f} \frac{\varepsilon}{2} \max \left(\left\|\zeta_{k} \widehat{Q}_{k}\right\|_{2},\left\|\zeta_{k}^{-1} \widehat{Q}_{k}^{-*}\right\|_{2}\right),
\end{aligned}
$$

where $\varepsilon$ is the machine epsilon and $d_{1}, d_{2}=d_{b}, d_{3}$, and $d_{f}$ are some modest constants that may depend on $n$, the details of the arithmetic and the inversion algorithm but depend neither on $Q_{k}$ nor $\widehat{Q}_{k}$. Each $Q_{k}$ is a smooth function of $Q_{k-1}$ and each $F_{k, j}=O(\varepsilon)$, so, by induction,

$$
\widehat{Q}_{k}=Q_{k}+O(\varepsilon) .
$$

Hence, the bounds above may be loosely expressed in terms of $Q_{k}$ as

$$
\begin{aligned}
\left\|F_{k b}\right\|_{2} & \leq d_{b} \varepsilon\left\|Q_{k}\right\|_{2}+O\left(\varepsilon^{2}\right) \\
\left\|F_{k f}\right\|_{2} & \leq d_{f} \frac{\varepsilon}{2} \max \left(\left\|\zeta_{k} Q_{k}\right\|_{2},\left\|\zeta_{k}^{-1} Q_{k}^{-*}\right\|_{2}\right)+O\left(\varepsilon^{2}\right) \\
& \leq d_{f} \varepsilon\left\|Q_{k+1}\right\|_{2}+O\left(\varepsilon^{2}\right) .
\end{aligned}
$$

Inequality (4.4) is a consequence of (2.2).

We need the following lemma for continuing our analysis.

Lemma 4.3. Let $A \in \mathbb{C}^{n \times n}$ be a nonsingular matrix with polar decomposition $A=Q H$, with $Q \in \mathbb{C}^{n \times n}$ unitary and $H \in \mathbb{C}^{n \times n}$ Hermitian positive definite. If $F \in \mathbb{C}^{n \times n}$ with $\|F\|_{2}=1$ and $t \geq 0$, then when $t$ is sufficiently small, $A+t F$ has the polar decomposition

$$
A+t F=Q\left((I+t E)+O\left(t^{2}\right)\right)\left(H+t G+O\left(t^{2}\right)\right),
$$

where $G \in \mathbb{C}^{n \times n}$ is the unique Hermitian solution to

$$
F^{*} A+A^{*} F=G H+H G,
$$

and $E \in \mathbb{C}^{n \times n}$ is the unique skew-Hermitian solution to

$$
Q^{*} F-F^{*} Q=E H+H E .
$$

Also, $E$ is given by

$$
E=\left(Q^{*} F-G\right) H^{-1}
$$

Proof. See proof in Appendix A.2.

At each of the perturbed Newton iteration (4.1) rounding errors are equivalent to perturbing $\widehat{Q}_{k}$ to $\widehat{Q}_{k}+F_{k b}$, taking one Newton step (1.1), then perturbing the result by adding $F_{k f}$. Let $\widehat{Q}_{k}=W_{k} \widehat{H}_{k}$ and $\widehat{Q}_{k}+F_{k b}=\widetilde{W}_{k} \widetilde{H}_{k}$ be the polar decompositions of $\widehat{Q}_{k}$ and $\widehat{Q}_{k}+F_{k b}$, respectively. By Lemma 4.3,

$$
\widetilde{W}_{k}=W_{k}\left(I+E_{k b}\right)+O\left(\varepsilon^{2}\right),
$$

Copyright $@$ by SIAM. Unauthorized reproduction of this article is prohibited. 
where $E_{k b}$ satisfies

$$
E_{k b} \widehat{H}_{k}+\widehat{H}_{k} E_{k b}=W_{k}^{*} F_{k b}-F_{k b}^{*} W_{k}+O\left(\varepsilon^{2}\right) .
$$

From (4.1),

$$
\frac{\zeta_{k}}{2}\left(\widehat{Q}_{k}+F_{k b}\right)+\frac{1}{2 \zeta_{k}}\left(\widehat{Q}_{k}+F_{k b}\right)^{-*}=\widehat{Q}_{k+1}-F_{k f} .
$$

Since the unitary factor in the polar decomposition of the left-hand side matrix is $\widetilde{W}_{k}$, applying Lemma 4.3 to $\widehat{Q}_{k+1}$, we have

$$
\widetilde{W}_{k}=W_{k+1}\left(I-E_{k f}\right)+O\left(\varepsilon^{2}\right),
$$

or equivalently,

$$
W_{k+1}=\widetilde{W}_{k}\left(I+E_{k f}\right)+O\left(\varepsilon^{2}\right)
$$

where $E_{k f}$ satisfies

$$
E_{k f} \widehat{H}_{k+1}+\widehat{H}_{k+1} E_{k f}=W_{k+1}^{*} F_{k f}-F_{k f}^{*} W_{k+1}+O\left(\varepsilon^{2}\right) .
$$

Since $Q_{k}$ has the polar decomposition $Q_{k}=Q H_{k}$ and $\widehat{Q}_{k}$ satisfies (4.2), by Lemma 4.3 one also has $\widehat{H}_{k}=H_{k}+O(\varepsilon), W_{k}=Q+O(\varepsilon)$. Based on these first order error results, (4.9) and (4.11) can be expressed as

$$
\begin{aligned}
E_{k b} H_{k}+H_{k} E_{k b} & =Q^{*} F_{k b}-F_{k b}^{*} Q+O\left(\varepsilon^{2}\right), \\
E_{k f} H_{k+1}+H_{k+1} E_{k f} & =Q^{*} F_{k f}-F_{k f}^{*} Q+O\left(\varepsilon^{2}\right) .
\end{aligned}
$$

Combining (4.8) and (4.10), one has

$$
W_{k+1}=W_{k}\left(I+E_{k b}+E_{k f}\right)+O\left(\varepsilon^{2}\right) .
$$

It follows by induction (with $W_{0}=Q$ ) that

$$
W_{j}=Q\left(I+E_{j}\right)+O\left(\varepsilon^{2}\right), \quad j>0,
$$

where

$$
E_{j}=\sum_{k=0}^{j-1}\left(E_{k b}+E_{k f}\right) .
$$

Suppose that Algorithm 3.1 applied to a nonsingular matrix $A \in \mathbb{C}^{n \times n}$, with polar decomposition $A=Q H$ completes after $p$ iterations in Step 1. We obtain $\widehat{Q}_{p}$ that satisfies $\left\|\widehat{Q}_{p}-\widehat{Q}_{p}^{-*}\right\|_{2} \leq\left\|\widehat{Q}_{p}-\widehat{Q}_{p}^{-*}\right\|_{F}<\delta$. With the polar decomposition $\widehat{Q}_{p}=W_{p} \widehat{H}_{p}$ and (2.2), we have (see the proof in Appendix A.3)

$$
\frac{1}{2}\left(\widehat{Q}_{p}+\widehat{Q}_{p}^{-*}\right)=W_{p}+\Delta \widehat{Q}_{p}
$$

where

$$
\left\|\Delta \widehat{Q}_{p}\right\|_{2}<\delta^{2} / 8
$$

Copyright $@$ by SIAM. Unauthorized reproduction of this article is prohibited. 
Suppose $\delta$ is small. Step 2 of the algorithm produces approximate polar factors

$$
\widehat{Q}=\frac{1}{2}\left(\widehat{Q}_{p}+\widehat{Q}_{p}^{-*}\right)+F, \quad \widehat{H}=\frac{1}{2}\left(\widehat{Q}^{*} A+A^{*} \widehat{Q}+K\right),
$$

where $F$ accounts for rounding error forming $\widehat{Q}$ from $\widehat{Q}_{p}$ and $K$ for rounding error forming $\widehat{H}$ from $A$ and $\widehat{Q}$ obeying

$$
\|F\| \leq d_{F} \varepsilon, \quad\|K\| \leq d_{K} \varepsilon\|A\|_{2},
$$

with modest constants $d_{F}$ and $d_{K}$. So we have

$$
\left\|\widehat{Q}-W_{p}\right\|_{2} \leq d_{F} \varepsilon+\delta^{2} / 8 .
$$

Since $W_{p}=Q(I+E)$ with $E:=E_{p}$ defined in (4.14), by (4.15),

$$
\widehat{Q}=Q(I+E)+\Delta \widehat{Q}_{p}+F=Q(I+E+L),
$$

where $L=Q^{*}\left(\Delta \widehat{Q}_{p}+F\right)$ satisfies

$$
\|L\|_{2} \leq d_{L} \max \left(\varepsilon, \delta^{2}\right)
$$

for some modest constant $d_{L}$, which combines the rounding error $F$ and the effect of stopping criterion. Both $d_{L}$ and $d_{K}$ may depend on $n$ and the details of the finite precision arithmetic and computational algorithm, but not on $A, \widehat{Q}$, or $\widehat{H}$. With (4.17) and the fact that $E$ is skew-Hermitian,

$$
\begin{aligned}
\widehat{H} & =\frac{1}{2}\left((I+E+L)^{*} Q^{*} A+A^{*} Q(I+E+L)+K\right) \\
& \left.=\frac{1}{2}\left(1-E+L^{*}\right) H+H(I+E+L)+K\right) \\
& =\frac{1}{2}\left(2 H-E H+H E+L^{*} H+H L+K\right) .
\end{aligned}
$$

So by (4.17) and (4.18), to the first order,

$$
\begin{aligned}
\widehat{Q} \widehat{H}-A= & \frac{1}{2} Q(I+E+L)\left(2 H-E H+H E+L^{*} H+H L+K\right)-A \\
= & \frac{1}{2} Q\left(2 H-E H+H E+L^{*} H+H L+2 E H+2 L H+K\right)-A \\
& +O\left(\|L\|_{2}^{2}\right)+O\left(\varepsilon\|L\|_{2}\right)+O\left(\varepsilon^{2}\right) \\
= & \frac{1}{2} Q\left(E H+H E+L^{*} H+H L+2 L H+K\right)+O\left(\max \left(\varepsilon^{2}, \varepsilon \delta^{2}, \delta^{4}\right)\right) .
\end{aligned}
$$

From (4.14) this expression can be written

$$
\begin{aligned}
\widehat{Q} \widehat{H}-A= & \frac{1}{2} Q \sum_{k=0}^{p-1}\left(E_{k b} H+H E_{k b}+E_{k f} H+H E_{k f}\right) \\
& +\frac{1}{2} Q\left(L^{*} H+H L+2 L H+K\right)+O\left(\max \left(\varepsilon^{2}, \varepsilon \delta^{2}, \delta^{4}\right)\right) .
\end{aligned}
$$

Note that so far the suboptimal scale factors have not played a role. In order to continue the analysis, we need the following lemma which involves the suboptimal scaling.

Copyright $@$ ( ) by SIAM. Unauthorized reproduction of this article is prohibited. 
Lemma 4.4. Let $A \in \mathbb{C}^{n \times n}$ be a nonsingular matrix with singular value decomposition $U \Sigma V^{*}, \Sigma=\operatorname{diag}\left(\sigma_{1}, \sigma_{2}, \ldots, \sigma_{n}\right)$, and $\sigma_{1} \geq \sigma_{2} \geq \cdots \geq \sigma_{n}$. Consider Newton's iteration (2.1) with scaling (1.6) and initial iterate $\Sigma_{0}=\Sigma$. Let $\sigma_{j}^{(k)}$ be the jth diagonal entry of $\Sigma_{k}$, and let $\sigma_{\max }^{(k)}=\max _{1 \leq j \leq n} \sigma_{j}^{(k)}$. If $a, b$ satisfy $0<a \leq \sigma_{n}$ and $b \geq \sigma_{1}$, then, for all $k \geq 0$ and $1 \leq i, j \leq n$,

$$
\frac{\sigma_{\max }^{(k)}}{\sigma_{i}^{(k)}+\sigma_{j}^{(k)}} \leq \frac{b}{\sigma_{i}+\sigma_{j}} .
$$

Proof. See Appendix A.4.

Recall that $E_{k b}, E_{k f}$ satisfy (4.12) and (4.13), respectively. Let

$$
H_{k}=V \Sigma_{k} V^{*}
$$

be an eigen-decomposition, where $V$ is unitary and $\Sigma_{k}$ is diagonal obeying (2.1). (Recall that throughout the algorithm the singular vectors of the $Q_{k}$ 's are the singular vectors of $A$. In particular, for all $k$, the unitary matrix of the right singular vectors of $A$ is also a unitary matrix of the eigenvectors of $H_{k}$.) In this notation, (4.12) and (4.13) can be written

$$
\begin{aligned}
\widetilde{E}_{k b} \Sigma_{k}+\Sigma_{k} \widetilde{E}_{k b} & =\widetilde{F}_{k b}-\widetilde{F}_{k b}^{*}+O\left(\varepsilon^{2}\right), \\
\widetilde{E}_{k f} \Sigma_{k+1}+\Sigma_{k+1} \widetilde{E}_{k f} & =\widetilde{F}_{k f}-\widetilde{F}_{k f}^{*}+O\left(\varepsilon^{2}\right),
\end{aligned}
$$

where

(4.20) $\widetilde{E}_{k b}=V^{*} E_{k b} V, \widetilde{E}_{k f}=V^{*} E_{k f} V, \widetilde{F}_{k b}=V^{*} Q^{*} F_{k b} V, \widetilde{F}_{k f}=V^{*} Q^{*} F_{k f} V$.

So, the $(i, j)$ th entries of $\widetilde{E}_{k b}$ and $\widetilde{E}_{k f}$ are

$$
\tilde{e}_{i j, k b}=\frac{\widetilde{f}_{i j, k b}-\overline{\widetilde{f}_{j i, k b}}}{\sigma_{i}^{(k)}+\sigma_{j}^{(k)}}+O\left(\varepsilon^{2}\right), \quad \widetilde{e}_{i j, k f}=\frac{\widetilde{f}_{i j, k f}-\overline{\widetilde{f}_{j i, k f}}}{\sigma_{i}^{(k+1)}+\sigma_{j}^{(k+1)}}+O\left(\varepsilon^{2}\right) .
$$

Note that $\left\|\widetilde{E}_{k j}\right\|_{2}=\left\|E_{k j}\right\|_{2}$ and $\left\|\widetilde{F}_{k j}\right\|_{2}=\left\|F_{k j}\right\|_{2}$ for $j=b, f$, because $V$ and $Q$ are unitary.

Multiplying (4.19) on the left by $V^{*}$ and on the right by $V$ gives

$$
\begin{aligned}
V^{*}(\widehat{Q} \widehat{H}-A) V= & \frac{1}{2} V^{*} Q V \sum_{k=0}^{p-1}\left(\widetilde{E}_{k b} \Sigma+\Sigma \widetilde{E}_{k b}+\widetilde{E}_{k f} \Sigma+\Sigma \widetilde{E}_{k f}\right) \\
& +\frac{1}{2} V^{*}\left(L^{*} H+H L+2 L H+K\right) V+O\left(\max \left(\varepsilon^{2}, \varepsilon \delta^{2}, \delta^{4}\right)\right),
\end{aligned}
$$

where $\widetilde{E}_{k b}$ and $\widetilde{E}_{k f}$ are given by (4.20). From (4.21), the $(i, j)$ th entry of the sum $\sum_{k=0}^{p-1}\left(\widetilde{E}_{k b} \Sigma+\Sigma \widetilde{E}_{k b}+\widetilde{E}_{k f} \Sigma+\Sigma \widetilde{E}_{k f}\right)$ is

$$
\begin{aligned}
& \sum_{k=0}^{p-1}\left(\widetilde{e}_{i j, k b}+\widetilde{e}_{i j, k f}\right)\left(\sigma_{i}+\sigma_{j}\right) \\
& =\sum_{k=0}^{p-1}\left(\frac{\widetilde{f}_{i j, k b}-\widetilde{\widetilde{f}_{j i, k b}}}{\sigma_{i}^{(k)}+\sigma_{j}^{(k)}}+\frac{\widetilde{f}_{i j, k f}-\widetilde{f}_{j i, k f}}{\sigma_{i}^{(k+1)}+\sigma_{j}^{(k+1)}}\right)\left(\sigma_{i}+\sigma_{j}\right)+O\left(\varepsilon^{2}\right) \\
& =\sum_{k=0}^{p-1}\left(\frac{\widetilde{f}_{i j, k b}-\overline{\widetilde{f}_{j i, k b}}}{\sigma_{\max }^{(k)}} \frac{\left(\sigma_{i}+\sigma_{j}\right) \sigma_{\max }^{(k)}}{\sigma_{i}^{(k)}+\sigma_{j}^{(k)}}+\frac{\widetilde{f}_{i j, k f}-\overline{\widetilde{f}_{j i, k f}}}{\sigma_{\max }^{(k+1)}} \frac{\left(\sigma_{i}+\sigma_{j}\right) \sigma_{\max }^{(k+1)}}{\sigma_{i}^{(k+1)}+\sigma_{j}^{(k+1)}}\right) \\
& \quad+O\left(\varepsilon^{2}\right) .
\end{aligned}
$$

Copyright $@$ by SIAM. Unauthorized reproduction of this article is prohibited. 
Inequalities (4.3) and (4.4) and Lemma 4.4 imply

$$
\begin{aligned}
& \left|\sum_{k=0}^{p-1}\left(\widetilde{e}_{i j, k b}+\widetilde{e}_{i j, k f}\right)\left(\sigma_{i}+\sigma_{j}\right)\right| \\
& \leq 2 \varepsilon \sum_{k=0}^{p-1}\left(d_{b} \frac{\left(\sigma_{i}+\sigma_{j}\right) \sigma_{\max }^{(k)}}{\sigma_{i}^{(k)}+\sigma_{j}^{(k)}}+d_{f} \frac{\left(\sigma_{i}+\sigma_{j}\right) \sigma_{\max }^{(k+1)}}{\sigma_{i}^{(k+1)}+\sigma_{j}^{(k+1)}}\right)+O\left(\varepsilon^{2}\right) \\
& \leq 2 p \varepsilon\left(d_{b}+d_{f}\right) b+O\left(\varepsilon^{2}\right) .
\end{aligned}
$$

Hence, the residual is bounded as

$$
\begin{gathered}
\|\widehat{Q} \widehat{H}-A\|_{2} \leq\left\|Q \sum_{k=0}^{p-1}\left(E_{k b} H+H E_{k b}+E_{k f} H+H E_{k f}\right) / 2\right\|_{2} \\
+\left\|Q\left(L^{*} H+H L+2 L H+K\right) / 2\right\|_{2}+O\left(\max \left(\varepsilon^{2}, \varepsilon \delta^{2}, \delta^{4}\right)\right) \\
\leq n p \varepsilon\left(d_{b}+d_{f}\right) b+\left(2 d_{L}+d_{K} / 2\right) \max \left(\varepsilon, \delta^{2}\right)\|A\|_{2}+O\left(\max \left(\varepsilon^{2}, \varepsilon \delta^{2}, \delta^{4}\right)\right) .
\end{gathered}
$$

In the same way, from (4.18) we can obtain

$$
\begin{aligned}
\|\widehat{H}-H\|_{2} & \leq\|(-E H+H E) / 2\|_{2}+\left\|\left(L^{*} H+H L+K\right) / 2\right\|_{2}+O\left(\delta^{4}\right) \\
& \leq n p \varepsilon\left(d_{b}+d_{f}\right) b+\left(d_{L}+d_{K} / 2\right) \max \left(\varepsilon, \delta^{2}\right)\|A\|_{2}+O\left(\max \left(\varepsilon^{2}, \varepsilon \delta^{2}, \delta^{4}\right)\right) .
\end{aligned}
$$

By applying Lemma 4.4 to (4.21) to estimate $\|E\|_{2}$, then from (4.17) we can derive

$$
\|\widehat{Q}-Q\|_{2} \leq\|Q E\|_{2}+\|Q L\|_{2} \leq \theta n p \varepsilon\left(d_{b}+d_{f}\right) b+d_{L} \max \left(\varepsilon, \delta^{2}\right),
$$

where

$$
\theta= \begin{cases}\frac{1}{\sigma_{n}} & A \text { is complex } \\ \frac{\sigma_{n-1}+\sigma_{n}}{n} & A \text { is real. }\end{cases}
$$

(The formula in the real case is based on the fact that $\widetilde{e}_{i i, k b}=\widetilde{e}_{i i, k f}=0$ from (4.21).)

We present the above error analysis results as well as (4.16) in the following theorem.

TheOREM 4.5. Suppose that $A \in \mathbb{C}^{n \times n}$ satisfies Assumption 4.2 and has the polar decomposition $A=Q H$. Let $\widehat{Q}$ and $\widehat{H}$ be the matrices computed by Algorithm 3.1 after $p$ iterations with a matrix inversion method that satisfies the error model in Assumption 4.1. Then

$$
\begin{aligned}
\|\widehat{Q} \widehat{H}-A\|_{2} & \leq n p \varepsilon\left(d_{b}+d_{f}\right) b+\left(2 d_{L}+d_{K} / 2\right) \max \left(\varepsilon, \delta^{2}\right)\|A\|_{2}+O\left(\max \left(\varepsilon^{2}, \varepsilon \delta^{2}, \delta^{4}\right)\right) \\
\|\widehat{H}-H\|_{2} & \leq n p \varepsilon\left(d_{b}+d_{f}\right) b+\left(d_{L}+d_{K} / 2\right) \max \left(\varepsilon, \delta^{2}\right)\|A\|_{2}+O\left(\max \left(\varepsilon^{2}, \varepsilon \delta^{2}, \delta^{4}\right)\right) \\
\|\widehat{Q}-Q\|_{2} & \leq \theta n p \varepsilon\left(d_{b}+d_{f}\right) b+d_{L} \max \left(\varepsilon, \delta^{2}\right) \\
\left\|\widehat{Q}-W_{p}\right\|_{2} & \leq d_{F} \varepsilon+\delta^{2} / 8
\end{aligned}
$$

where $d_{b}, d_{f}, d_{L}, d_{K}, d_{F}$ are some modest constants, $W_{p}$ is a unitary matrix, and $\theta$ is defined in (4.22).

As noted in Table 2.1, in most practical situations $p \leq 9$. Therefore, if $b$ is not too much greater than $\|A\|_{2}$ and the algorithm uses a stopping criterion $\delta$ not too much greater than $\sqrt{\varepsilon}$, then the algorithm is backward stable.

Copyright (c) by SIAM. Unauthorized reproduction of this article is prohibited. 
COROLlary 4.6. If $b=\|A\|_{2}$ and $\delta=n^{\frac{1}{4}} \sqrt{\varepsilon}$, then

$$
\begin{aligned}
\|\widehat{Q} \widehat{H}-A\|_{2} & \leq\left(n p\left(d_{b}+d_{f}\right)+\sqrt{n}\left(2 d_{L}+d_{K} / 2\right)\right) \varepsilon\|A\|_{2}+O\left(\varepsilon^{2}\right) \\
\|\widehat{H}-H\|_{2} & \leq\left(n p\left(d_{b}+d_{f}\right)+\sqrt{n}\left(d_{L}+d_{K} / 2\right)\right) \varepsilon\|A\|_{2}+O\left(\varepsilon^{2}\right) \\
\|\widehat{Q}-Q\|_{2} & \leq n p\left(d_{b}+d_{f}\right) \varepsilon\left(\theta\|A\|_{2}\right)+\sqrt{n} d_{L} \varepsilon \\
\left\|\widehat{Q}-W_{p}\right\|_{2} & \leq\left(d_{F}+\sqrt{n} / 8\right) \varepsilon .
\end{aligned}
$$

Note that the error bounds for $\|\widehat{H}-H\|_{2}$ and $\|\widehat{Q}-Q\|_{2}$ coincide with the perturbation results; see, for example, $[8,21,20]$ and $[11$, section 8.2$]$. The quantity $\theta\|A\|_{2}$ serves as the condition number for the perturbation of $Q$.

Remark 1. The same procedure can be used to give an error analysis for Newton's method with other scalings. Note that the backward stability depends on whether

$$
\frac{\left(\sigma_{i}+\sigma_{j}\right) \sigma_{\max }^{(k)}}{\sigma_{i}^{(k)}+\sigma_{j}^{(k)}}=O\left(\|A\|_{2}\right),
$$

which depends on scaling factors. From Remark 2 in Appendix A.4, (4.23) holds for the optimal scaling (1.2). Lemma A.3 in Appendix A.5 shows that (4.23) also holds for the $(1, \infty)$-scaling (1.3) and the scaling (1.4). Therefore, Newton's method with these three scalings is also backward stable under the same conditions of Theorem 4.5 and an appropriate stopping criterion, when the number of iterations is not too large. (See Remark 3 in Appendix A.5.)

We also observed that the numerical stability doesn't necessarily depend on how fast the method converges. In fact, one can show that when $\|A\|_{2} \geq\left\|A^{-1}\right\|_{2}$, Newton's method without scaling $(a=b=1)$ computes a polar decomposition satisfying the same error bounds given in Theorem 4.5.

5. Numerical examples. We did some numerical experiments with Newton's method (1.1) with scaling (1.6) using $a=\left\|A^{-1}\right\|_{F}^{-1}, b=\|A\|_{F}$, and also with the $(1, \infty)$-scaling (1.3). The main purpose is to test the numerical stability results and convergence rate and to compare the suboptimal scaling and $(1, \infty)$-scaling. For this reason we used the bidiagonal reduction matrix inversion method Algorithm BR for computing matrix inverses.

All numerical experiments were done on a Dell personal computer with a PentiumIV processor, in MATLAB version 7.2 with machine epsilon $\varepsilon \approx 2.22 \times 10^{-16}$.

In the numerical experiments we use stopping criterion $\delta=n^{\frac{1}{4}} \sqrt{\varepsilon}$, where $n$ is the size of matrices. For Newton's method with the $(1, \infty)$-scaling, the scale factor is shifted to 1 when $\left\|X_{k+1}-X_{k}\right\|_{F} /\left\|X_{k+1}\right\|_{F}<10^{-2}$. Based on the results in Corollary 4.6, if $\widehat{Q}$ and $\widehat{H}$ are the computed unitary and Hermitian polar factors produced by Algorithm 3.1, then we expect to observe that $\|A-\widehat{Q} \widehat{H}\|_{2} /\|A\|_{2},\|H-\widehat{H}\|_{2} /\|H\|_{2}$, and $\|Q-\widehat{Q}\|_{2} /\left(\theta\|A\|_{2}\right)$ are not much larger than $\varepsilon$.

In the tables we will use the following notations:

$$
e_{Q}=\frac{\|Q-\widehat{Q}\|_{2}}{\theta\|A\|_{2}}, \quad e_{H}=\frac{\|H-\widehat{H}\|_{2}}{\|H\|_{2}}, \quad \text { res }=\frac{\|A-\widehat{Q} \widehat{H}\|_{2}}{\|A\|_{2}}, \quad \text { ror }=\left\|\widehat{Q}^{*} \widehat{Q}-I\right\|_{2},
$$

where the "exact" factors $Q$ and $H$ for the matrices in the first example are obtained from the SVD of $A$ using MATLAB's variable precision arithmetic vpa with 24 signi- 
TABLE 5.1

The extreme values of errors, residuals, and iteration counts from Example 1.

\begin{tabular}{c|c|cc|cc|cc}
\hline \multicolumn{2}{c|}{ kappa } & \multicolumn{2}{c|}{$10^{2}$} & \multicolumn{2}{c|}{$10^{8}$} & \multicolumn{2}{c}{$10^{15}$} \\
\hline \multicolumn{2}{l|}{} & Min & Max & Min & Max & Min & Max \\
\hline$p$ & SUB & 6 & 6 & 8 & 8 & 8 & 9 \\
& HSF & 6 & 7 & 8 & 8 & 8 & 9 \\
\hline$e_{Q}$ & SUB & $2.6 e-17$ & $7.2 e-17$ & $7.4 e-19$ & $1.7 e-17$ & $6.9 e-19$ & $2.3 e-17$ \\
& HSF & $2.2 e-17$ & $7.7 e-17$ & $7.4 e-19$ & $1.7 e-17$ & $6.9 e-19$ & $2.3 e-17$ \\
\hline$e_{H}$ & SUB & $2.2 e-16$ & $4.1 e-16$ & $2.5 e-16$ & $4.1 e-16$ & $1.9 e-16$ & $4.4 e-16$ \\
& HSF & $1.7 e-16$ & $3.9 e-16$ & $1.9 e-16$ & $3.9 e-16$ & $2.2 e-16$ & $4.0 e-16$ \\
\hline$r e s$ & SUB & $4.1 e-16$ & $8.9 e-16$ & $4.0 e-16$ & $7.5 e-16$ & $3.5 e-16$ & $6.3 e-16$ \\
& HSF & $3.5 e-16$ & $8.2 e-16$ & $2.7 e-16$ & $5.9 e-16$ & $3.9 e-16$ & $7.2 e-16$ \\
\hline ror & SUB & $7.9 e-16$ & $1.1 e-15$ & $8.0 e-16$ & $1.1 e-15$ & $7.8 e-16$ & $1.3 e-15$ \\
& HSF & $7.0 e-16$ & $1.2 e-15$ & $7.9 e-16$ & $1.2 e-15$ & $8.2 e-16$ & $1.1 e-15$ \\
\hline
\end{tabular}

TABLE 5.2

Errors, residuals, and iteration counts for Hilbert matrices from Example 2.

\begin{tabular}{c|c|ccccc}
\hline \multicolumn{2}{l|}{$n$} & 6 & 8 & 10 & 12 & 14 \\
\hline$p$ & SUB & 8 & 8 & 9 & 9 & 9 \\
& HSF & 7 & 8 & 8 & 9 & 9 \\
\hline$e_{Q}$ & SUB & $1.2 e-18$ & $1.6 e-19$ & $2.7 e-19$ & $4.1 e-19$ & $2.0 e-17$ \\
& HSF & $1.2 e-18$ & $1.6 e-19$ & $2.7 e-19$ & $4.1 e-19$ & $2.0 e-17$ \\
\hline$e_{H}$ & SUB & $2.3 e-16$ & $1.9 e-16$ & $8.7 e-17$ & $1.4 e-16$ & $2.3 e-16$ \\
& HSF & $1.9 e-16$ & $1.4 e-16$ & $8.7 e-17$ & $1.6 e-16$ & $2.7 e-16$ \\
\hline$r e s$ & SUB & $2.6 e-16$ & $2.4 e-16$ & $1.8 e-16$ & $3.0 e-16$ & $3.8 e-16$ \\
& HSF & $2.5 e-16$ & $2.5 e-16$ & $1.8 e-16$ & $3.3 e-16$ & $6.3 e-16$ \\
\hline$r o r$ & SUB & $2.6 e-16$ & $3.9 e-16$ & $6.2 e-16$ & $6.3 e-16$ & $6.5 e-16$ \\
& HSF & $2.8 e-16$ & $5.3 e-16$ & $6.8 e-16$ & $8.5 e-16$ & $1.0 e-15$ \\
\hline
\end{tabular}

ficant decimal digits. $p$ is the number of iterations, and $n$ is the dimension of matrices. The symbol "HSF" refers to Newton's method (1.1) with Higham's $(1, \infty)$-scaling. The symbol "SUB" refers to (1.1) with scaling (1.6) using the Frobenius norms for the initial interval, i.e., $a=\left\|A^{-1}\right\|_{F}^{-1}$ and $b=\|A\|_{F}$.

Example 1. Three groups of twenty real matrices were constructed with dimension 20 by using MATLAB's gallery ('randsvd' , 20,kappa,5), with kappa equal to $10^{2}, 10^{8}, 10^{15}$, respectively. The singular values of the generated matrices are random values with uniformly distributed logarithm. For each group the ranges of the condition numbers $\kappa_{2}(A)$ and $\theta\|A\|_{2}$ are listed below:

$$
\begin{aligned}
& \text { kappa }=10^{2}: \kappa_{2}(A) \in[37.7,90.6], \theta\|A\|_{2} \in[33.5,83.7] \\
& \text { kappa }=10^{8}: \kappa_{2}(A) \in[1.13,6.75] \times 10^{7}, \theta\|A\|_{2} \in[0.2,5.44] \times 10^{7}, \\
& \text { kappa }=10^{15}: \kappa_{2}(A) \in\left[6.35 \times 10^{10}, 7.53 \times 10^{14}\right], \theta\|A\|_{2} \in\left[1.78 \times 10^{10}, 5.22 \times 10^{14}\right] .
\end{aligned}
$$

The test results are summarized in Table 5.1, where, for each group, the minimum and maximum values of the errors, residuals, and numbers of iterations are listed.

Example 2. In this example the test matrices are the Hilbert matrices, which are $n \times n$ matrices with entries $a_{i j}=1 /(i+j-1)$. The example uses dimensions $n=6$, $n=8, n=10, n=12$, and $n=14$. For every Hilbert matrix, the polar decomposition is $A_{n}=I_{n} A_{n}$.

The condition number $\kappa_{2}\left(A_{n}\right)$ ranges from $1.5 \times 10^{7}$ to $5.1 \times 10^{17}$, and $\theta\left\|A_{n}\right\|_{2}$ ranges from $2.6 \times 10^{5}$ to $1.0 \times 10^{17}$. The test results are reported in Table 5.2.

Newton's method with the suboptimal scaling performed well in both examples calculating the polar factors to nearly full precision. As predicted it takes at most 9 iterations. Newton's method with the $(1, \infty)$-scaling performs equally well. 
6. Conclusion. The suboptimal scaling scheme (1.6) is essentially costless and simplifies the algorithm of Newton's iteration (1.1) for computing polar factors. In a typical floating point system, with this scaling scheme, for matrices with $\kappa_{2}(A)<10^{16}$, no more than 9 iterations are needed for convergence to the unitary polar factor with a convergence tolerance roughly equal to the machine epsilon. By employing the bidiagonal factorization for matrix inversion, (1.1) with (1.6) forms a provably backward stable algorithm. Newton's method with $(1, \infty)$-scaling and scaling (1.4) is also proved to be backward stable, provided the number of iterations is not too large.

\section{Appendix A.}

\section{A.1. Bidiagonal reduction-based matrix inversion algorithm.} Algorithm BR.

Input: Nonsingular matrix $A \in \mathbb{C}^{n \times n}$

Output: $G=A^{-1}$

Step 1: Compute $A=U B V^{*}$, with $U, V$ unitary and $B$ upper bidiagonal.

Step 2: Solve $B Y=U^{*}$ for $Y$ by back substitution.

Step 3: Compute $G=V Y$.

In Step 1 one may use the Householder reflectors to perform the reduction. The reduction needs $\frac{8}{3} n^{3}$ flops and computing $U$ needs $\frac{4}{3} n^{3}$ flops. The matrix $V$ is stored in factorized form. The cost for solving the matrix equation is $O\left(n^{2}\right)$ flops. With the factorized form of $V$, it needs $2 n^{3}$ flops to compute $G$. So the total cost is $6 n^{3}$ flops.

In order to show that a matrix inverse computed by Algorithm BR follows Assumption 4.1, we need the following lemma.

Lemma A.1. Consider the system $B x=z$, where $z \in \mathbb{C}^{n}$ and $B$ is nonsingular and upper bidiagonal denoted by

$$
B=\left[\begin{array}{cccc}
\alpha_{1} & \beta_{1} & & 0 \\
& \ddots & \ddots & \\
& & \ddots & \beta_{n-1} \\
0 & & & \alpha_{n}
\end{array}\right] .
$$

Let $\hat{x}$ be the numerical solution with back substitution. Then $\hat{x}$ satisfies

$$
\hat{x}=B^{-1}(z+\delta z)+\delta x,
$$

where

$$
|\delta z| \leq 3 n \varepsilon|z|+O\left(\varepsilon^{2}\right), \quad|\delta x| \leq 3 n \varepsilon|\hat{x}|+O\left(\varepsilon^{2}\right) .
$$

Proof. The components of the computed vector $\hat{x}$ can be formulated as

$$
\hat{x}_{n}=\frac{z_{n}}{\alpha_{n}\left(1+\epsilon_{n}\right)}, \quad \hat{x}_{k}=\frac{z_{k}\left(1+\delta_{k}\right)-\beta_{k} \hat{x}_{k+1}}{\alpha_{k}\left(1+\epsilon_{k}\right)}, \quad 1 \leq k \leq n-1,
$$

where $\left|\epsilon_{n}\right|,\left|\delta_{k}\right|<\varepsilon,\left|\epsilon_{k}\right|<3 \varepsilon, k \leq n-1$. So we have

$$
\begin{aligned}
& \alpha_{n} \hat{x}_{n}\left(1+\epsilon_{n}\right)=z_{n} \\
& \alpha_{n-1} \hat{x}_{n-1}\left(1+\epsilon_{n-1}\right)+\beta_{n-1} \hat{x}_{n}=z_{n-1}\left(1+\delta_{n-1}\right) \\
& \vdots \\
& \alpha_{1} \hat{x}_{1}\left(1+\epsilon_{1}\right)+\beta_{1} \hat{x}_{2}=z_{1}\left(1+\delta_{1}\right) .
\end{aligned}
$$

Copyright (C) by SIAM. Unauthorized reproduction of this article is prohibited. 
Define $\tilde{x}=\left[\tilde{x}_{1}, \ldots, \tilde{x}_{n}\right]^{T}, \tilde{z}=\left[\tilde{z}_{1}, \ldots, \tilde{z}_{n}\right]^{T}$, with

$$
\begin{aligned}
& \tilde{x}_{n}=\hat{x}_{n}\left(1+\epsilon_{n}\right), \quad \tilde{x}_{n-1}=\hat{x}_{n-1}\left(1+\epsilon_{n-1}\right)\left(1+\epsilon_{n}\right), \quad \ldots, \quad \tilde{x}_{1}=\hat{x}_{1} \prod_{k=1}^{n}\left(1+\epsilon_{k}\right), \\
& \tilde{z}_{n}=z_{n}, \quad \tilde{z}_{n-1}=z_{n-1}\left(1+\delta_{n-1}\right)\left(1+\epsilon_{n}\right), \quad \ldots, \quad \tilde{z}_{1}=z_{1}\left(1+\delta_{1}\right) \prod_{k=2}^{n}\left(1+\epsilon_{k}\right) .
\end{aligned}
$$

By multiplying $\left(1+\epsilon_{n}\right)$ to the second equation, $\left(1+\epsilon_{n}\right)\left(1+\epsilon_{n-1}\right)$ to the third equation, and so on in (A.1), we obtain that $\tilde{x}$ and $\tilde{z}$ satisfy

$$
B \tilde{x}=\tilde{z} .
$$

Let $\delta z=\tilde{z}-z$, and $\delta x=\hat{x}-\tilde{x}$. Then from $\tilde{x}=B^{-1} \tilde{z}$ we have

$$
\hat{x}=B^{-1}(z+\delta z)+\delta x .
$$

The error bounds for $|\delta x|$ and $|\delta z|$ follow simply from the definitions.

Theorem A.2. Let $X$ be the inverse of A computed by Algorithm BR. Then $X$ satisfies Assumption 4.1.

Proof. We only consider the first order errors.

Let $\hat{U} \hat{B} \hat{V}^{*}$ be the computed bidiagonal factorization of $A$. Then $\hat{U}=U+\Delta U_{1}$, $\hat{V}=V+\Delta V_{1}$, where $U, V$ are unitary and $\left\|\Delta U_{1}\right\|_{2} \leq d_{1} \varepsilon,\left\|\Delta V_{1}\right\|_{2} \leq d_{2} \varepsilon$, and

$$
U \hat{B} V^{*}=A+E,
$$

where $\|E\|_{2} \leq d_{3} \varepsilon\|A\|_{2}$ for some modest constants $d_{1}, d_{2}, d_{3}$. Let $\hat{Y}$ be the numerical solution of $\hat{B} Y=\hat{U}^{*}$ computed by back substitution. By Lemma A.1,

$$
\hat{Y}=\hat{B}^{-1}\left(\hat{U}^{*}+\Delta U_{2}\right)+\Delta Y,
$$

where

$$
\left\|\Delta U_{2}\right\|_{2} \leq 3 n^{\frac{3}{2}} \varepsilon, \quad\|\Delta Y\|_{2} \leq 3 n^{\frac{3}{2}} \varepsilon\|\hat{Y}\|_{2} .
$$

Let $X$ be the computed matrix product $\hat{V} \hat{Y}$. We have

$$
X=\hat{V} \hat{Y}+\Delta X
$$

where $\|\Delta X\|_{2} \leq d_{4} \varepsilon\|\hat{Y}\|_{2}$ for some modest constant $d_{4}$. Now

$$
\begin{aligned}
X & =\hat{V} \hat{B}^{-1}\left(\hat{U}^{*}+\Delta U_{2}\right)+\hat{V} \Delta Y+\Delta X=\hat{V} \hat{B}^{-1} \hat{U}^{*}+\hat{V} \hat{B}^{-1} \Delta U_{2}+\hat{V} \Delta Y+\Delta X \\
& =: V \hat{B}^{-1} U^{*}+F=(A+E)^{-1}+F,
\end{aligned}
$$

where

$$
F=\Delta V_{1} \hat{B}^{-1} U^{*}+V \hat{B}^{-1}\left(\Delta U_{1}\right)^{*}+\hat{V} \hat{B}^{-1} \Delta U_{2}+\hat{V} \Delta Y+\Delta X .
$$

It is easily verified that $\|F\|_{F} \leq\left(6 n^{\frac{3}{2}}+d_{1}+d_{2}+d_{4}\right) \varepsilon\left\|A^{-1}\right\|_{2}$. 
A.2. Proof of Lemma 4.3. Equations (4.5) and (4.7) are established in the proof of Theorem 2.5 in [8]. Here we slightly modify that proof to establish (4.6).

Let $A(t)=A+t F$ have polar decompositions $Q(t) H(t)$. Note that $H(t)=$ $\left(A(t)^{*} A(t)\right)^{1 / 2}$ (positive definite square root) and $Q(t)=A(t) H(t)^{-1}$ are sums, differences, products, quotients, and compositions with $C^{\infty}$ functions of the entries of $A(t)$, which is trivially a $C^{\infty}$ function. Hence, $Q(t)$ and $H(t)$ are also $C^{\infty}$. (Here we use the fact that $A$ is nonsingular to observe that $H(t)=\left(A(t)^{*} A(t)\right)^{1 / 2}$ avoids the singularity of the square root at zero.) Using $\dot{Q}$ and $\dot{H}$ to denote differentiation by $t$, Taylor's theorem implies that

$$
\begin{aligned}
& Q(t)=Q(0)+t \dot{Q}(0)+O\left(t^{2}\right)=Q\left(I+t Q^{*} \dot{Q}(0)\right)+O\left(t^{2}\right) \\
& H(t)=H(0)+t \dot{H}(0)+O\left(t^{2}\right) .
\end{aligned}
$$

The proof of Theorem 2.5 in [8] shows $\dot{H}(0)=G=G^{*}$, with $G$ given by (4.5) and $Q^{*}(0) \dot{Q}(0)=E=-E^{*}$, with $E$ given by $(4.7)$.

Differentiate $A+t F=Q(t) H(t)$ to get $F=\dot{Q} H+Q \dot{H}$. Evaluating at $t=0$, letting $E=Q^{*}(0) \dot{Q}(0), G=\dot{H}(0)$ gives $Q^{*} F=E H+G$. Using the facts that $E$ is skew-Hermitian and $G$ is Hermitian while subtracting this equation to its Hermitian transpose gives (4.6). The Lyapunov operator on the right is nonsingular, because $A$ nonsingular implies that the eigenvalues of the Hermitian polar factor $H$ are real and positive. Hence, the solution $E$ is unique.

A.3. Proof for (4.15). Let $\widehat{Q}_{p}=U_{p} \Sigma_{p} V_{p}^{*}$ be the SVD. Recall that the singular values satisfy $\sigma_{i}^{(p)} \geq 1$. Then $\left\|\widehat{Q}_{p}-\widehat{Q}_{p}^{-*}\right\|_{2}<\delta$ implies that

$$
\sigma_{i}^{(p)}-\frac{1}{\sigma_{i}^{(p)}}<\delta, \quad i=1,2, \ldots, n
$$

Because

$$
\sigma_{i}^{(p)}-\frac{1}{\sigma_{i}^{(p)}}=\frac{\left(\sigma_{i}^{(p)}+1\right)\left(\sigma_{i}^{(p)}-1\right)}{\sigma_{i}^{(p)}}
$$

we have

$$
\sigma_{i}^{(p)}-1<\frac{\delta \sigma_{i}^{(p)}}{\sigma_{i}^{(p)}+1}
$$

Then

$$
\frac{1}{2}\left(\sigma_{i}^{(p)}+\frac{1}{\sigma_{i}^{(p)}}\right)-1=\frac{\left(\sigma_{i}^{(p)}-1\right)^{2}}{2 \sigma_{i}^{(p)}}<\frac{\sigma_{i}^{(p)}}{2\left(\sigma_{i}^{(p)}+1\right)^{2}} \delta^{2} .
$$

Since the function $x /(x+1)^{2}$ is decreasing when $x \geq 1$, we have $\sigma_{i}^{(p)} /\left(\sigma_{i}^{(p)}+1\right)^{2} \leq 1 / 4$. Hence

$$
\frac{1}{2}\left(\sigma_{i}^{(p)}+\frac{1}{\sigma_{i}^{(p)}}\right)-1<\delta^{2} / 8
$$

and using $W_{p}=U_{p} V_{p}^{*}$,

$$
\begin{aligned}
\left\|\Delta \widehat{Q}_{p}\right\|_{2} & =\left\|\left(\widehat{Q}_{p}+\widehat{Q}_{p}^{-*}\right) / 2-W_{p}\right\|_{2}=\left\|U_{p}\left(\left(\Sigma_{p}+\Sigma_{p}^{-1}\right) / 2-I\right) V_{p}^{*}\right\|_{2} \\
& =\max _{i}\left(\frac{1}{2}\left(\sigma_{i}^{(p)}+\frac{1}{\sigma_{i}^{(p)}}\right)-1\right)<\delta^{2} / 8 .
\end{aligned}
$$

Copyright $\odot$ by SIAM. Unauthorized reproduction of this article is prohibited. 
A.4. Proof of Lemma 4.4. By (2.4), $\sigma_{\max }^{(k)} \leq b_{k}$ for $k \geq 0$.

So we only need to show that

$$
\frac{b_{k}}{\sigma_{i}^{(k)}+\sigma_{j}^{(k)}} \leq \frac{b}{\sigma_{i}+\sigma_{j}}, \quad k \geq 0 .
$$

An easy calculation shows, for all $i$ and $j$,

$$
\sigma_{i}^{(k)}+\sigma_{j}^{(k)}=\frac{\zeta_{k-1}}{2}\left(\sigma_{i}^{(k-1)}+\sigma_{j}^{(k-1)}\right)\left(1+\frac{1}{\zeta_{k-1}^{2} \sigma_{i}^{(k-1)} \sigma_{j}^{(k-1)}}\right) .
$$

Recall $b_{k}$ satisfies

$$
b_{k}=\frac{1}{2}\left(\zeta_{k-1} b_{k-1}+\frac{1}{\zeta_{k-1} b_{k-1}}\right)=\frac{\zeta_{k-1}}{2} b_{k-1}\left(1+\frac{1}{\zeta_{k-1}^{2} b_{k-1}^{2}}\right) .
$$

Because $\sigma_{i}^{(k-1)} \sigma_{j}^{(k-1)} \leq b_{k-1}^{2}$,

$$
\begin{aligned}
\frac{b_{k}}{\sigma_{i}^{(k)}+\sigma_{j}^{(k)}} & =\frac{\frac{\zeta_{k-1}}{2} b_{k-1}\left(1+\frac{1}{\zeta_{k-1}^{2} b_{k-1}^{2}}\right)}{\frac{\zeta_{k-1}}{2}\left(\sigma_{i}^{(k-1)}+\sigma_{j}^{(k-1)}\right)\left(1+\frac{1}{\zeta_{k-1}^{2} \sigma_{i}^{(k-1)} \sigma_{j}^{(k-1)}}\right)} \\
& \leq \frac{b_{k-1}}{\sigma_{i}^{(k-1)}+\sigma_{j}^{(k-1)}} .
\end{aligned}
$$

An easy induction on $k$ now implies that

$$
\frac{b_{k}}{\sigma_{i}^{(k)}+\sigma_{j}^{(k)}} \leq \frac{b_{0}}{\sigma_{i}^{(0)}+\sigma_{j}^{(0)}}=\frac{b}{\sigma_{i}+\sigma_{j}}, \quad k \geq 0 .
$$

Remark 2.

1. The condition $a \leq\left\|A^{-1}\right\|_{2}^{-1}$ is essential for proving Lemma 4.4. Without it one may not have the inequality $\sigma_{j}^{(k)} \leq b_{k}$, and the result cannot be proved.

2. In the case that the optimal scaling is employed, $b_{k}=\sigma_{\max }^{(k)}$, and one has the same result.

\section{A.5. Relation (4.23) for the scalings (1.3) and (1.4).}

Lemma A.3. Suppose that the matrix sequence $Q_{k}$ is generated by Newton's iteration with scaling $\zeta_{k}$ that is either $\zeta_{k}^{(1, \infty)}$ in (1.3) or $\zeta_{k}^{(F)}$ in (1.4). The singular values of $Q_{k}$ satisfy

$$
\frac{\sigma_{\max }^{(k)}}{\sigma_{i}^{(k)}+\sigma_{j}^{(k)}} \leq\left(\prod_{\ell=0}^{k-1} \psi_{\ell}\right) \frac{\|A\|_{2}}{\sigma_{i}+\sigma_{j}} \leq n^{\frac{k}{2}} \frac{\|A\|_{2}}{\sigma_{i}+\sigma_{j}}, \quad k \geq 0, \quad 1 \leq i, j \leq n,
$$

where $\psi_{\ell}=\max \left\{1, \frac{1}{\zeta_{\ell}^{2} \sigma_{\max }^{(\ell)} \sigma_{\min }^{(\ell)}}\right\} \leq \sqrt{n}$.

Proof. Let

$$
w_{k}=\frac{1}{\zeta_{k}^{2} \sigma_{\min }^{(k)} \sigma_{\max }^{(k)}}, \quad k \geq 0
$$

Copyright $@$ by SIAM. Unauthorized reproduction of this article is prohibited. 
If

$$
\zeta_{k}=\zeta_{k}^{(1, \infty)}=\sqrt[4]{\frac{\left\|Q_{k}^{-1}\right\|_{1}\left\|Q_{k}^{-1}\right\|_{\infty}}{\left\|Q_{k}\right\|_{1}\left\|Q_{k}\right\|_{\infty}}}
$$

using the inequalities $\|A\|_{2} \leq \sqrt{\|A\|_{1}\|A\|_{\infty}} \leq \sqrt{n}\|A\|_{2}$, we have ([11, p. 208])

$$
\frac{1}{\sqrt[4]{n}} \zeta_{k} \leq \frac{1}{\sqrt{\sigma_{\min }^{(k)} \sigma_{\max }^{(k)}}} \leq \sqrt[4]{n} \zeta_{k} .
$$

If

$$
\zeta_{k}=\zeta_{k}^{(F)}=\sqrt{\frac{\left\|Q_{k}^{-1}\right\|_{F}}{\left\|Q_{k}\right\|_{F}}}
$$

using the inequalities $\|A\|_{2} \leq\|A\|_{F} \leq \sqrt{n}\|A\|_{2}$, we also have (A.3).

So in both cases we have

$$
\frac{1}{\sqrt{n}} \leq w_{k} \leq \sqrt{n}
$$

Construct an interval $\left[a_{k}, b_{k}\right]$ according to the rule

$$
\begin{array}{lll}
a_{k}=\sigma_{\min }^{(k)} w_{k}, & b_{k}=\sigma_{\max }^{(k)}, & w_{k} \leq 1, \\
a_{k}=\sigma_{\min }^{(k)}, & b_{k}=\sigma_{\max }^{(k)} w_{k}, & w_{k} \geq 1 .
\end{array}
$$

Because $a_{k} \leq \sigma_{\min }^{(k)}$ and $b_{k} \geq \sigma_{\max }^{(k)}$, we have $\sigma_{1}^{(k)}, \ldots, \sigma_{n}^{(k)} \in\left[a_{k}, b_{k}\right]$. Also, in both cases we have $\left(\zeta_{k} a_{k}\right)^{-1}=\zeta_{k} b_{k}$. So $\rho\left(\zeta_{k} a_{k}\right)=\rho\left(\zeta_{k} b_{k}\right)$ and

$$
\sigma_{j}^{(k+1)}=\rho\left(\zeta_{k} \sigma_{j}^{(k)}\right) \in \rho\left(\zeta_{k}\left[a_{k}, b_{k}\right]\right)=\left[1, \rho\left(\zeta_{k} b_{k}\right)\right], \quad j=1,2, \ldots, n .
$$

Since

$$
\rho\left(\zeta_{k} b_{k}\right)=\frac{1}{2}\left(\zeta_{k} b_{k}+\frac{1}{\zeta_{k} b_{k}}\right)=\frac{\zeta_{k} b_{k}}{2}\left(1+\frac{1}{\left(\zeta_{k} b_{k}\right)^{2}}\right)
$$

we have

$$
\begin{aligned}
\frac{\sigma_{\max }^{(k+1)}}{\sigma_{i}^{(k+1)}+\sigma_{j}^{(k+1)}} & \leq \frac{\rho\left(\zeta_{k} b_{k}\right)}{\sigma_{i}^{(k+1)}+\sigma_{j}^{(k+1)}}=\frac{\frac{\zeta_{k} b_{k}}{2}\left(1+\frac{1}{\left(\zeta_{k} b_{k}\right)^{2}}\right)}{\frac{\zeta_{k}}{2}\left(\sigma_{i}^{(k)}+\sigma_{j}^{(k)}\right)\left(1+\frac{1}{\zeta_{k}^{2} \sigma_{i}^{(k)} \sigma_{j}^{(k)}}\right)} \\
& =\frac{b_{k}\left(1+\frac{1}{\left(\zeta_{k} b_{k}\right)^{2}}\right)}{\left(\sigma_{i}^{(k)}+\sigma_{j}^{(k)}\right)\left(1+\frac{1}{\zeta_{k}^{2} \sigma_{i}^{(k)} \sigma_{j}^{(k)}}\right)} .
\end{aligned}
$$

If $w_{k} \leq 1$, then $b_{k}=\sigma_{\max }^{(k)}$. Because $\sigma_{i}^{(k)} \sigma_{j}^{(k)} \leq\left(\sigma_{\max }^{(k)}\right)^{2}=b_{k}^{2}$, we have

$$
\frac{\sigma_{\max }^{(k+1)}}{\sigma_{i}^{(k+1)}+\sigma_{j}^{(k+1)}} \leq \frac{b_{k}}{\sigma_{i}^{(k)}+\sigma_{j}^{(k)}}=\frac{\sigma_{\max }^{(k)}}{\sigma_{i}^{(k)}+\sigma_{j}^{(k)}} .
$$

Copyright $@$ by SIAM. Unauthorized reproduction of this article is prohibited. 
If $w_{k} \geq 1$, then $b_{k}=\sigma_{\max }^{(k)} w_{k}$. Because $\sigma_{i}^{(k)} \sigma_{j}^{(k)} \leq\left(\sigma_{\max }^{(k)}\right)^{2} \leq b_{k}^{2}$, we have

$$
\frac{\sigma_{\max }^{(k+1)}}{\sigma_{i}^{(k+1)}+\sigma_{j}^{(k+1)}} \leq \frac{b_{k}}{\sigma_{i}^{(k)}+\sigma_{j}^{(k)}}=w_{k} \frac{\sigma_{\max }^{(k)}}{\sigma_{i}^{(k)}+\sigma_{j}^{(k)}} .
$$

Hence

$$
\frac{\sigma_{\max }^{(k+1)}}{\sigma_{i}^{(k+1)}+\sigma_{j}^{(k+1)}} \leq \psi_{k} \frac{\sigma_{\max }^{(k)}}{\sigma_{i}^{(k)}+\sigma_{j}^{(k)}}, \quad \psi_{k}=\max \left\{1, w_{k}\right\} \leq \sqrt{n} .
$$

Then the inequalities in (A.2) can be easily derived.

Remark 3. Suppose that Newton's method with scaling $\zeta_{k}^{(1, \infty)}$ or $\zeta_{k}^{(F)}$ terminates after $p$ iterations. We have the same error bounds as in Theorem 4.5 but with a factor $n^{\frac{p}{2}}$ in the first term of the bounds for $\|\widehat{Q} \widehat{H}-A\|_{2},\|\widehat{H}-H\|_{2}$, and $\|\widehat{Q}-Q\|_{2}$. When $n$ and $p$ are both large, this factor is notably large, and one may not be able to use the bounds to claim backward stability. Unfortunately, we are unable to provide an upper bound for $p$, although it is observed that $p$ is usually moderate in practice ( $p \leq 9$ for the $(1, \infty)$-scaling for all examples in section 5$)$.

However, we argue that the factor $n^{\frac{p}{2}}$ is an overestimate. The point is that, when $Q_{k}$ is getting close to a unitary matrix, $\zeta_{k}^{(1, \infty)}$ and $\zeta_{k}^{(F)}$ are getting close to 1 . Then $w_{k}$ as well as $\psi_{k}$ will be close to 1 . So in practice $w_{k}$ will be around 1 after a couple of iterations.

Acknowledgments. We thank Nick Higham for detailed suggestions and sending part of his unpublished book [11]. We thank Andrzej Kiełbasiński for pointing out a mistake in an earlier version and the referees for their comments and suggestions.

\section{REFERENCES}

[1] Z. Bai and J. Demmel, Design of a parallel nonsymmetric eigenroutine toolbox, Part I, in Proceedings of the Sixth SIAM Conference on Parallel Processing for Scientific Computing, R. F. Sincovec et al., eds., SIAM, Philadelphia, 1993, pp. 391-398. Also available as Computer Science Report CSD-92-718, University of California, Berkeley, CA 1992.

[2] Z. Bai and J. Demmel, Design of a Parallel Nonsymmetric Eigenroutine Toolbox, Part II, Technical report Department of Mathematics Research Report 95-11, University of Kentucky, Lexington, KY, 1995.

[3] L. A. BALzer, Accelerated convergence of the matrix sign function method of solving Lyapunov, Riccati and other matrix equations, Internat. J. Control, 32 (1980), pp. 1057-1078.

[4] R. Byers, Solving the algebraic Riccati equation with the matrix sign function, Linear Algebra Appl., 85 (1987), pp. 267-279.

[5] A. A. Dubrulle, An optimum iteration for the matrix polar decomposition, Electron. Trans. Numer. Anal., 8 (1999), pp. 21-25 (electronic).

[6] W. Gander, Algorithms for the polar decomposition, SIAM J. Sci. Statist. Comput., 11 (1990), pp. $1102-1115$.

[7] G. H. Golub and C. F. Van LoAn, Matrix Computations, Johns Hopkins Studies in the Mathematical Sciences, 3rd ed., Johns Hopkins University Press, Baltimore, MD, 1996.

[8] N. J. Higham, Computing the polar decomposition-with applications, SIAM J. Sci. Statist. Comput., 7 (1986), pp. 1160-1174.

[9] N. J. Higham, Computing a nearest symmetric positive semidefinite matrix, Linear Algebra Appl., 103 (1988), pp. 103-118.

[10] N. J. Higham, Accuracy and Stability of Numerical Algorithms, 2nd ed., SIAM, Philadelphia, 2002.

[11] N. J. Higham, Functions of Matrices: Theory and Computation, SIAM, Philadelphia, 2008.

[12] N. J. Higham And P. Papadimitriou, Parallel Singular Value Decomposition via the Polar Decomposition, Technical report Numerical Analysis Report 239, Department of Mathematics, University of Manchester, Manchester, England, 1993.

Copyright $@$ by SIAM. Unauthorized reproduction of this article is prohibited. 
[13] N. J. Higham and P. Papadimitriou, A parallel algorithm for computing the polar decomposition, Parallel Comput., 20 (1994), pp. 1161-1173.

[14] N. J. Higham And R. S. Schreiber, Fast polar decomposition of an arbitrary matrix, SIAM J. Sci. Statist. Comput., 11 (1990), pp. 648-655.

[15] R. A. Horn and C. R. Johnson, Matrix Analysis, Cambridge University Press, Cambridge, 1990, corrected reprint of the 1985 original.

[16] C. S. Kenney and A. J. Laub, On scaling Newton's method for polar decomposition and the matrix sign function, SIAM J. Matrix Anal. Appl., 13 (1992), pp. 688-706.

[17] C. S. Kenney and A. J. Laub, The matrix sign function, IEEE Trans. Automat. Control, 40 (1995), pp. 1330-1348.

[18] A. KiębasińSKi AND K. ZiȨTAK, Numerical behavior of Higham's scaled method for polar decomposition, Numer. Algorithms, 32 (2003), pp. 105-140.

[19] A. Kiębasiński, P. Zieliński, and K. Ziȩtak, Higham's scaled method for polar decomposition and numerical matrix-inversion, Technical report Institute of Mathematics and Computer Science Report 118/2007/P-045, Wroclaw University of Technology, Wroclaw, Poland, 2007.

[20] R.-C. LI, New perturbation bounds for the unitary polar factor, SIAM J. Matrix Anal. Appl., 16 (1995), pp. 327-332.

[21] R. Mathias, Perturbation bounds for the polar decomposition, SIAM J. Matrix Anal. Appl., 14 (1993), pp. 588-597.

[22] J. D. RoberTs, Linear model reduction and solution of the algebraic Riccati equation by use of the sign function, Internat. J. Control, 32 (1980), pp. 677-687. (Reprint of Technical report TR-13, CUED/B-Control, Cambridge University, Engineering Department, Cambridge, 1971).

[23] P. Zieliński And K. Ziģtak, Polar decomposition-properties, applications and algorithms, Appl. Math., An. Polish Math. Soc., 38 (1995), pp. 23-49.

Copyright (C) by SIAM. Unauthorized reproduction of this article is prohibited. 